\title{
Meso-Linked Multiporphyrins as Model for Light Harvesting Systems: Review
}

\section{Anchal Singhal*}

Department of Chemistry, University of Delhi, Delhi, India

\begin{abstract}
Nature has always been the fundamental source of inspiration for researchers to understand the complex biological functions. Photosynthesis is among the important natural phenomena and hence, gained interest towards developing the artificial photosynthetic reaction centers. Electron and energy transfer process between various porphyrin units within the multiporphyrin arrays is the important key in developing such systems. Long duration of charge separated state and spectral coverage scope of the accepting porphyrin units are another important factor that contribute to an efficient mimic of the photosynthetic reaction center. Meso-linked porphyrin architectures provide a great aspect in this regard because of short inter-porphyrinic distance with optimal dihedral angle. The present review highlights the design of various kinds of meso-linked multiporphyrins and study of electron and energy transfer processes between them that serve as an efficient model for light harvesting systems.
\end{abstract}

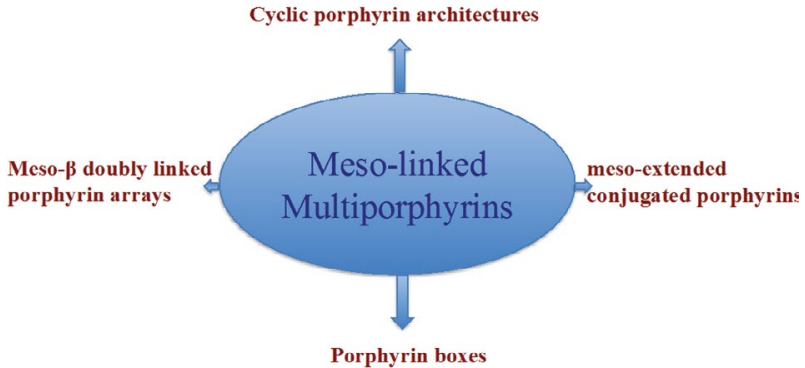

Keywords: Multiporphyrins; Meso-linked porphyrin; Cyclic arrays; Electron and energy transfer; Electronic coupling; Light harvesting system

\section{Introduction}

Nature is often the ultimate goal for chemists. Photosynthesis is one of the most important natural processes. Nature, over the billions of years, possesses the molecular structures to achieve this process of photosynthesis. During photosynthesis, plants convert light energy into electrochemical energy and eventually into chemical potential energy stored in carbohydrates and other compounds. The carbohydrates are oxidized to provide energy to the living organism. The importance of photosynthesis has driven many researchers to look for ways to duplicate the fundamental features of photosynthesis in simplified systems. In designing systems for light energy conversion, nature utilizes multicomponent arrays of organic molecules to channel the energy and electron transfer processes in the light harvesting antenna and photosynthetic reaction centers. The structure, orientations and interactions between the constituent units of the arrays of organic molecules determine the energy and electron transfer processes in biological systems.

Nature has designed a most sophisticated molecular system for the reaction center in order to achieve effective charge separation. Primary energy transfer and trapping in photosynthesis occurs in sophisticated machinery consisting of a so-called antenna and a reaction center (RC) together forming a photosynthetic unit (PSU). The antenna is an array of pigment molecules, which absorbs light energy and transfers it in a form of molecular electronic excitation to a trap, the RC, where primary charge separation takes place. In nature, many different types of RCs and even more antennas exist in the form of chlorophylls, Quinone's, carotenoid polyenes, and many other organic molecules. The light harvesting in purple bacteria is among the most studied and best understood processes of primary photosynthesis that helps in the development of artificial light harvesting systems [1].

The arrangement of bacterial photosynthetic system unit (PSU) was first analyzed by X-ray crystallography in 1995 [2-4]. The PSU of purple bacteria generally consists of more than one antenna complex. The peripheral antenna (LH2) is in touch with the core antenna (LH1), which surrounds the RC. The peripheral antenna LH2 consists of two concentric rings of $\mathrm{BChl}$ molecules named $\mathrm{B} 800$ and B850 according to their characteristic $\mathrm{Q}_{\mathrm{y}}$ absorption maxima at $800 \mathrm{~nm}$ and $850 \mathrm{~nm}$. B800 contains nine bacteriochlorophylls arranged in a planar circle form. In contrast, B850 contains 18 bacteriochlorophylls in a barrel form arranged perpendicularly to B800. The light energy absorbed by B800 is transferred to B850 according to the cascading energy transfer. The excitation energy travels further to other B850s and finally reaches LH1. Here, 30 bacteriochlorophylls B880s are arranged in a barrel form similar to B850, except for having one open exit as it lacks one pair of bacteriochlorophyll and does not form a full ring. In addition, LH1 contains the reaction center in the central space. Therefore, the excitation energy reaching LH1 is transferred effectively to the special pair initiating the charge separation. The arrangements of B850, LH1, and interestingly even the special pair are given by coordination of the imidazolyl side chains in transmembrane helices to the central $\mathrm{Mg}^{2+}$

*Corresponding author: Anchal Singhal, Department of Chemistry, University of Delhi, Delhi-110 007, India, Tel: +919818721809; E-mail: singhal.anchal@gmail.com

Received March 15, 2017; Accepted March 23, 2017; Published March 28, 2017

Citation: Singhal A (2017) Meso-Linked Multiporphyrins as Model for Light Harvesting Systems: Review. Nat Prod Chem Res 5: 259. doi: 10.4172/23296836.1000259

Copyright: ( 2017 Singhal A. This is an open-access article distributed under the terms of the Creative Commons Attribution License, which permits unrestricted use, distribution, and reproduction in any medium, provided the original author and source are credited. 
ion in the bacteriochlorophyll [5]. The energy transfer rates in the supramolecular arrangements are as fast as the order of picoseconds within lifetimes of the order of nanoseconds [6-10].

To mimic the natural system, a large number of studies have been devoted to create artificial, highly ordered arrays of chromophores with the ultimate goal to construct devices that can efficiently capture light and utilize the excitation energy to transfer it to a designated acceptor point [11]. The highly efficient cooperation between the chromophore units is necessary for effective transfer of excitation energy, which has been achieved by connecting them in a well-defined manner by means of covalent bonds [12].

\section{Multiporphyrins as chemical models for photosynthesis}

Porphyrins comprise an important area of research interest in organic chemistry due to their potential in wide range of applications including light-harvesting arrays [13,14], molecular wires [15-18], photovoltaic cells [19], nonlinear optics [20-23], and photodynamic therapy [24]. Porphyrins are interesting in view of visible-light (Soret band around $400 \mathrm{~nm}$ and Q-band around 500-700 nm) absorbing and emitting materials as candidates for the study of electron and energy transfer processes to serve as artificial photosynthetic building units as well as for opto-electronic applications [25]. In order to construct multiporphyrins with well-defined shape and dimensions, numerous synthetic approaches have been developed. These approaches include covalent synthesis [26,27], and self-assembly by metal coordination [28-30] or hydrogen bonding [31-33]. Covalent linking is the most classical and productive method for the synthesis of such chemical models $[34,35]$.

In the covalently linked porphyrin arrays, a variety of linkages such as ethyne [36,37], polyyne [38-40], ethylene [41], alkane $[42,43]$ and aromatic entities [44-46] have been used to bridge the individual porphyrins. Lindsey et al. have reported the synthesis of numerous phenylene- and diphenyl ethynyl-bridged multiporphyrins for mimicking the biological light-harvesting antenna systems and their photo physical properties have been studied [46-48]. These multiporphyrins show efficient energy transfer that involves a throughbond process mediated by the bridge. However, these porphyrin arrays show very weak ground-state electronic interactions between the porphyrin units. Various linear types of meso- to meso acetyleneand butadiyne-bridged multiporphyrins have also been reported and their photo physical and electrochemical properties have been determined [36-38]. In contrast to the arene-bridged porphyrin arrays, these types of multiporphyrins exhibit strong ground- and excitedstate interporphyrin electronic coupling. The studies on these highly conjugated multiporphyrin arrays have provided significant insights into the nature of the interporphyrin interactions and invaluable information for rational design of porphyrin-based optoelectronic materials.

The wheel-like chromophore arrays play an essential role in the efficient capturing of light energy and its subsequent funneling to the reaction center [49]. These structural features have motivated chemists to design artificial light-harvesting antennae consisting of multiple porphyrin units and to explore their photochemical properties to generate a general approach for achieving the vectorial transfer of energy over a long distance to a designated point. Such synthetic lightharvesting antenna molecules contribute to a better understanding of the photochemical events involved in biological photosynthesis. The recent development of covalent (molecular) and noncovalent (supramolecular) approaches to the design of light-harvesting multiporphyrin arrays includes 1) ring-shaped [50-52], 2) star-shaped
$[53,54], 3)$ windmill-shaped $[55,56]$, and 4) dendritic architectures [57]. Their geometry-dependent photochemical properties are of great importance in artificial light harvesting systems.

The power of self-assembly synthesis lies in its ability to rapidly generate large and sophisticated molecular architectures from readily accessible building blocks with maximum efficiency. Extensive hands-on synthetic steps are minimized because the pathway to the formation of assemblies is guided by the nature of recognition surfaces programmed into the components. This provides access to high yields under thermodynamic control. A ligand displaying multiple, divergent Lewis basic sites provides a means to extend the self-assembly strategy to three-dimensional metal-templated porphyrin arrays. Metal-toligand interactions or hydrogen bonding between long porphyrin arrays was described by Branda and group as an efficient means to achieve the immense electronic communication through long distances between porphyrin moieties of supramoleclar structures [51].

\section{Electronic interactions between the porphyrins}

The electronic interactions of neighboring porphyrin chromophores in the arrays are the most important parameters for efficient electron transfer processes. The absorption spectrum reflects such kind of interactions among the porphyrins. The electronic interactions between neighboring porphyrins are changed when incorporated into a different geometry with different conformational freedom. The simple point dipole exciton coupling theory has been developed by Kasha et al. [58] which was found very useful to interpret the spectral changes caused by the inter-chromophore interactions, where the strength of the dipole interaction is represented by coulombic interactions that depend on the oscillator strength, orientation, and distance. The allowed lower energy transition ( $J$-type coupling) has been observed due to interaction of the transition dipole moments in a head-to-tail arrangement, while that parallel arrangement results in an allowed higher energy transition ( $H$-type coupling). The spectral changes observed in the Soret bands are due to exciton coupling, since the magnitude of exciton coupling is proportional to the square of oscillator strength. The components of the Soret band, Bx and By, which are degenerate in a porphyrin monomer, independently interact with the transition dipole moments of neighboring porphyrins but in a porphyrin dimer they couple differently. Excitonically coupled states are generated in electrostatically interacting porphyrins in a close arrangement. Transitions are allowed to lower the energy of two Bx states and the two unperturbed transitions By and Bz. Thus, the Soret band of porphyrin dimer splits into a red-shifted Bx component and unperturbed By, Bz components as only $\mathrm{Bx}$ transitions are parallel, and other dipole interactions of $\mathrm{By}$, $\mathrm{Bz}$ components have been cancelled out for an averaged perpendicular conformation of dimer product.

\section{Meso-meso linked porphyrin arrays}

Covalent multiporphyrin arrays are attracting interest as multichromophoric model systems for the study of electron transfer process in natural photosynthetic systems, as well as in the development of novel functional materials [13]. Various types of covalently linked arrays of metallo porphyrins have been designed and synthesized with the goal of applying these molecular oligomers to molecular photonic devices [26,52,59-62] as artificial light-harvesting systems [34,62-65]. Among them, meso-meso linked porphyrinic arrays have been emerged as an interesting candidate for exploring the artificial photosynthetic reaction centers and light harvesting antenna complexes (Figure 1). First rational synthesis of directly linked meso-porphyrinic arrays has been done by Susumu et al. in 1996 [66]. Since then, several approaches 


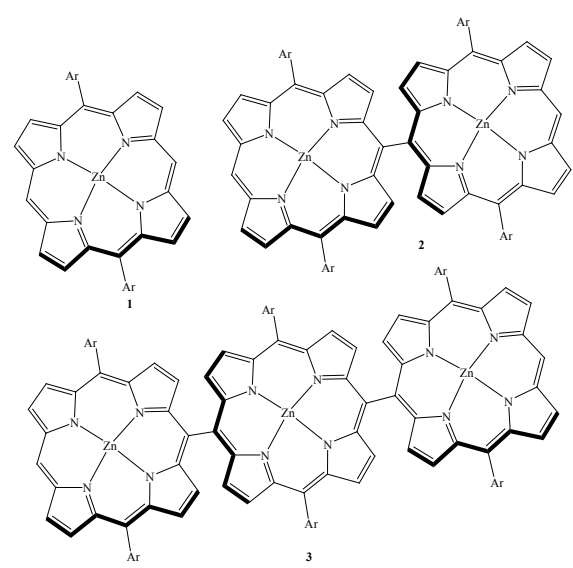

Figure 1: Meso-meso linked porphyrin arrays.

have been reported to prepare such meso-meso linked porphyrin arrays, such as Smith's condensation of dipyrromethane derivative with tetrakis (5-formyl-2-pyrrolyl)ethane [67], Osuka's oxidative dimerization of monomeric porphyrins, either chemically with silver salts [68] or electrochemically [69], Senge's oxidative dimerization of anionic adducts induced by 2,3-dichloro-5,6- dicyanoquinone (DDQ) [70] and Liebeskind's solvent-dependent DDQ-induced oxidative dimerization of zincate substituted porphyrins [71]. The organic solubility and architectural rigidity of covalently linked multiporphyrinic arrays are two important criteria for the systematic study of efficient electron and energy transfer processes and the porphyrinic pigments should be incorporated in precise states of metalation and geometrical arrangement. Meso-unsubstituted diaryl porphyrins are important precursors for the synthesis of meso-functionalized covalently linked porphyrin arrays, by the use of oxidative coupling reactions in the presence of silver (I) salts or by anodic electrochemical oxidation. The Ag (I) promoted meso-meso oxidative coupling reaction of 5,15-diaryl zinc (II) porphyrin has been used in the synthesis of a variety of porphyrin arrays such as linear, 3-D extended windmill arrays, dodecameric porphyrin wheel and various other interesting and useful directly linked porphyrin arrays [72,73].

A variety of porphyrin arrays has been reported by Osuka et al. by the use of coupling reactions in the presence of $\mathrm{Ag}$ (I) salts [74]. On treating the zinc porphyrin possessing unsubstituted meso positions with $\mathrm{Ag}$ (I) salt, the meso-meso linked diporphyrins and oligomeric porphyrins have been formed. This coupling reaction is highly regioselective as the reaction takes place selectively at the meso position of porphyrin monomer. The use of iodine in combination with silver (I) salts or iodine reagents has also been dictated in the literature for the formation of meso-meso linked porphyrin oligomers [72]. Various iodine (III) reagents such as $\mathrm{PhI}(\mathrm{O}-\mathrm{CO}-\mathrm{CF} 3) 2$ (PIFA, also named as bis[(trifluoroacetoxy)iodo]-benzene) or $\mathrm{PhI}(\mathrm{OAc}) 2$ (PIDA) were used for the coupling reactions of meso-porphyrins to form directly linked porhyrin dimers or oligomers [75].

\section{Meso- $\beta$ doubly linked porphyrin arrays}

Meso- $\beta$ doubly linked porphyrin arrays have been reported in the literature to be synthesized from oxidative oligomerization of a 5,15-diaryl $\mathrm{Ni}$ (II) porphyrin, in which $\mathrm{a}_{1 \mathrm{u}} \mathrm{HOMO}$ of $\mathrm{Ni}$ (II) plays a central role for coupling regio chemistry and thus the formation of meso- $\beta$ doubly linked porphyrin arrays have been limited to the use of nickel metalated porphyrin as precursors only. In the recent years, the synthesis of meso- $\beta$ doubly linked zinc metalated porphyrin dimer 7 and anti- and syn-trimers 8 and 9 has also been reported by Osuka et al. through DDQ-Sc(OTf) 3 oxidation of the corresponding meso- $\beta$ singly linked porphyrin precursors under the suitable reaction conditions (Scheme 1) [76]. Their precursors, the meso-meso linked porphyrin dimer 4 and trimers ( 5 and 6 ) have been synthesized by the Suzuki-Miyaura coupling reaction of $\beta$-borylated porphyrin, prepared from 5,15- bis(3,5-dioctyloxyphenyl)-10-(3,5-dimethylphenyl)substituted zinc(II) porphyrin, and mono meso-brominated porphyrin, prepared from 5,15-bis(3,5-dioctyloxyphenyl)-10-phenyl-substituted porphyrin. The $1 \mathrm{H}$ NMR spectra of zinc metalated meso- $\beta$ doubly linked porphyrin arrays exhibited sharper peaks than that of their nickel metalated analogues [77]. The meso- $\beta$ doubly linked porphyrin arrays having zinc as central metal atom found interesting applications due to their significant ground-state and excited-state electronic properties. Various hybrid arrays of meso-meso linked porphyrin[26] hexaphyrin-porphyrin along with their triply linked tapes have also been synthesized and their absorption spectrum was noticed near infra-red region [78].

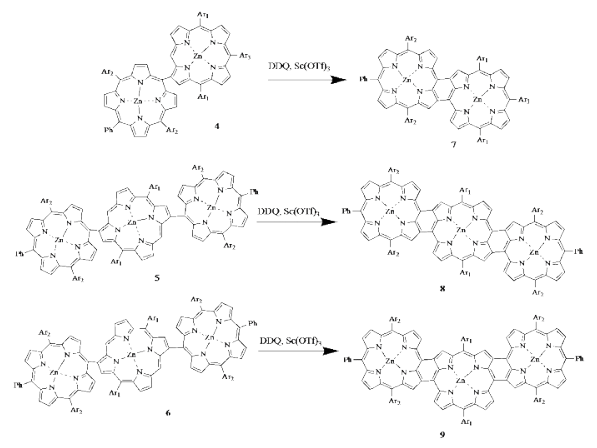

Scheme 1: Synthesis of meso- $\beta$ doubly linked porphyrin dimer and trimer

\section{Synthesis of meso-extended conjugated porphyrins}

Meso-extended porphyrins have been formed by the reaction of meso-tetraethynylporphyrins with aromatic halides in the presence of palladium complexes and due to extended conjugation, their absorption spectra have been bathochromically shifted [79]. The red-shifted electronic absorption spectra of meso-ethynylporphyrins, which resulted from efficient porphyrin-acetylene conjugation, make them attractive for applications in light harvesting systems and nonlinear optics [80]. Butadiyne linkers were also used for synthesis of meso-extended porphyrin oligomers as reported by Anderson et al. [80]. Such conjugated porphyrins exhibited maximum absorption bands around $900 \mathrm{~nm}$. Various modifications have been obtained using more soluble substrate such as 5,15-bis (3,5- di-tert-butylphenyl)10,20-bis(trihexylsilylethynyl)porphyrin with stepwise synthetic strategy including protodesilylation of TBAF and Glaser-Hay coupling conditions (Figure 2). The red shift in the absorption bands tend to be intensified with increase in chain length of meso-bridged butadiyne linkers. Peripheral extension seems to be a versatile approach for tuning the porphyrin absorption spectrum, and hence, various linkers such as ethene, ethyne, butadiyne etc. have been used to synthesize various porphyrin tapes and oligomers to serve efficient mimic of biological process of photosynthesis [81-83].

\section{Synthesis of core-modified meso-linked porphyrin arrays}

The modification of porphyrin core by replacing one or two inner nitrogens with other heteroatoms such as sulfur, oxygen, selenium, and tellurium forms a group of core-modified porphyrins containing different kinds of porphyrin cores such as $\mathrm{N}_{3} \mathrm{~S}, \mathrm{~N}_{2} \mathrm{~S}_{2}, \mathrm{~N}_{3} \mathrm{O}, \mathrm{N}_{2} \mathrm{SO}$, 
$\mathrm{N}_{2} \mathrm{OS}, \mathrm{N}_{3} \mathrm{Se}, \mathrm{N}_{3} \mathrm{Te}, \mathrm{N}_{2} \mathrm{Se}_{2}$, etc. [84]. The core-modified porphyrins exhibit interesting properties in terms of both aromatic character and their ability to stabilize metals in unusual oxidation states [85]. The electronic properties of core-modified porphyrins are quite different from normal porphyrins $\left(\mathrm{N}_{4}\right.$ core). Van Patten et al. [86] on the basis of computational studies predicted that a set of porphyrins such as $\mathrm{N}_{4}, \mathrm{~N}_{3} \mathrm{O}, \mathrm{N}_{3} \mathrm{~S}, \mathrm{~N}_{2} \mathrm{OS}$, and $\mathrm{N}_{2} \mathrm{~S}_{2}$ porphyrins arranged in a linear series with a progressive decrease in energy levels could provide the basis for an energy cascade. Various efforts have been directed in design and synthesis of unsymmetrical arrays containing two different macrocycles linked through meso-position such as porphyrin-chlorin [87], porphyrin-corrole [88], porphyrin-pheophorbide [89], and porphyrinphthalocyanine [90] macrocycles in the presence of palladium complexes, which are expected to have unusual electronic structure and interesting photophysical properties. These unsymmetrical arrays are useful to study singlet-singlet energy transfer and to obtain fast initial charge transfer and a slow back reaction, thus giving a long-lived charge-transfer state.

\section{Electron and energy transfer processes in directly linked diporphyrins}

The various types of diporphyrins such as TPP-type and OEP-type, bridged by the same conjugative spacers provide a nice opportunity to demonstrate and evaluate the important contribution of through bond-excitation energy transfer (TB-EET) in the overall energy transfer processes. Excitation energy transfer processes are the most important functions of antenna complexes. Thus, many artificial model compounds have been explored, which absorb visible light in a wide range and funnel the resulting excited state energy rapidly and efficiently to a designed site. There are two mechanisms for excitation energy transfer, Forster-type (through-space, TS) energy transfer by coulombic interaction between transition dipole moments and Dexter-type (through-bond, TB) energy transfer via electron-exchange interaction through direct or indirect overlap of the molecular orbitals. The importance of the orbital interaction on the TB-EET rate was exhibited by the comparison of tetraphenylporphyrin (TPP)-type diporphyrins versus octaethylporphyrin (OEP)-type diporphyrins, both of which have the same center-to center distance between the two porphyrin units [91]. The energy transfer rates in the TPP-type diporphyrins are distinctly larger as compared to their OEP-type counterparts but such transfer rate enhancement decreases on decreasing the distance between the two porphyrins [92]. This rate enhancement has been understood in terms of the significant contribution of TB-EET in TPP-type diporphyrin models. The TPP-type zinc porphyrins have an $\mathrm{a}_{2 \mathrm{u}} \mathrm{HOMO}$ with large electron densities at the meso-positions where unsaturated bridges are connected, while OEP-type zinc porphyrins have an $\mathrm{a}_{1 \mathrm{u}}$ HOMO with nodes at the meso-positions. Therefore, the TB orbital interactions are effective for TPP-type diporphyrins only.

In the $\beta, \beta$ '-substituted OEP-type porphyrins, the bulky peripheral alkyl substituents suppress the through-bond electronic interactions due to steric hindrance and thus, the meso-aryl bridges adopt perpendicular conformations with respect to the porphyrin plane. The enhanced contribution of the Forster mechanism for excitation energy transfer has been accounted when the bridging group between the two porphyrins becomes shorter, as the energy transfer rate enhancement decreased on decreasing the distance between the porphyrins as in case of TPP-type diporphyrins. The Forster excitation energy transfer is highly operative for a donor-acceptor (D-A) model with quite a short D-A separation [91]. On the contrary, the Forster energy transfer rate decreases quickly with increasing the distance between two porphyrin units. Thus, the through bond-excitation energy transfer (TB-EET) becomes predominant for diporphyrins with long distances between the porphyrins because of relatively small attenuation of through bondexcitation energy transfer (TB-EET) versus distance for diporphyrins with $\pi$-electronic bridges.

Various multi-porphyrin arrays have been constructed using several types of shorter linkers that are suitable for preparing linear or extended architectures via meso position attachment [92]. The overall orthogonal conformation between the adjacent porphyrin units in the orthogonal porphyrin arrays disrupts $\pi$-electron conjugation over the array despite of the very short inter-porphyrinic distance. Thus, the successful preparation of long porphyrinic arrays raises fundamental questions regarding exciton coupling, $\pi$-electron delocalization, and relative orientations between the adjacent porphyrins. The control of the dihedral angle of the meso-meso coupled diporphyrins is an intriguing factor for the systematic study of inter-porphyrinic interactions [9395], because it offers a fine-tuning of electronic interactions between the two porphyrins, which results in the manipulation of intramolecular excitation energy and electron transfer processes [96].

The electronic interactions between the adjacent porphyrin moieties in the meso-meso linked porphyrin arrays are minimum at perpendicular dihedral angle. Therefore, deviation of dihedral angle in the porphyrin ring causes a symmetry change from D2d to D2 with a simultaneous increase in the electronic interactions between the porphyrins as in the case of meso-meso linked diporphyrins strapped with a dioxymethylene group $\mathrm{Sn}$, where $\mathrm{n}$ is the number of carbon atoms in the chain (Figure 3 ). This change in symmetry alters the photophysical properties of porphyrinic arrays depending on the degree of electronic interactions between the porphyrins [97]. The directly linked orthogonal porphyrin arrays provide the prospects as artificial light harvesting arrays and molecular photonic wires because the unique photophysical aspect of these molecular arrays arising from substantial interchromophoric electronic interactions mimics the facile energy migration processes in biological light harvesting assemblies, where electronic delocalization is negligible due to a lack of direct bond linkage between individual pigment molecules [91].

\section{Non-covalent meso-linked cyclic porphyrin arrays}

The synthesis of covalently and non-covalently linked discrete cyclic porphyrin arrays as models of the photosynthetic light-harvesting antenna complexes has been well documented in the literature. A series of extremely long yet discrete meso-meso linked porphyrin arrays and covalently linked large porphyrin rings has been synthesized using silver(I)-promoted oxidative coupling strategy by Osuka et al. [98]. The photophysical properties of these molecules were studied using steadystate absorption, fluorescence, fluorescence lifetime, fluorescence
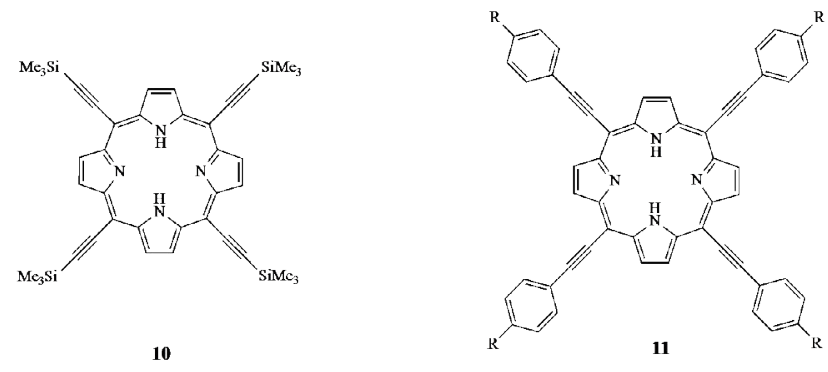

$\mathrm{R}-\mathrm{H}, \mathrm{Bu}, 3,5-\mathrm{L}-\mathrm{Bu}, \mathrm{C}_{\mathrm{g}} \mathrm{H}_{\mathrm{b}}$

Figure 2: Meso-ethynyl and meso-extended porphyrins 


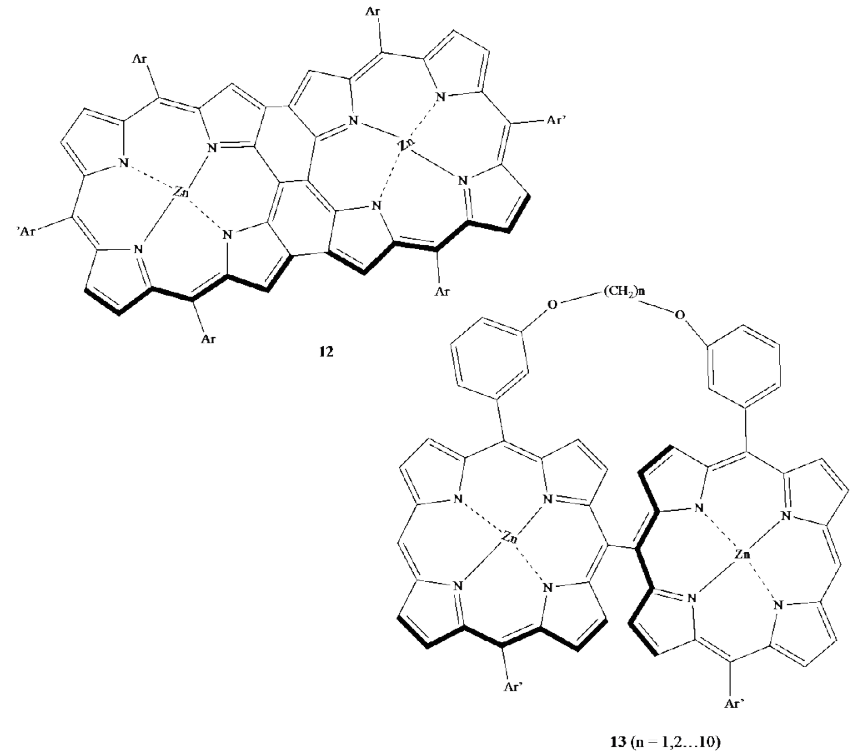

Figure 3: Various meso-meso linked porphyrins.

anisotropy decay, and transient absorption measurements. Both the transient absorption anisotropy decay profiles and the pump-power dependence on the femtosecond transient absorption are directly related to the EET processes within the porphyrin framework. The polarization anisotropy rise time and the exciton-exciton annihilation time are well defined within these structures in terms of the Forstertype incoherent energy-hopping model.

Meso-pyridine-linked zinc(II) porphyrins and their mesolinked dimers have been reported to get assembled spontaneously in the presence of non-coordinating solvents such as $\mathrm{CHCl}_{3}$ to form tetrameric porphyrin squares and porphyrin boxes, respectively [98]. In the porphyrin boxes, efficient EET throughout the cyclic porphyrin arrays was well recognized along with rigorous homochiral selfsorting process. The cyclic trimer has been reported to form mesocinchomeronimide appended zinc(II) porphyrin. It was also shown that the discrete cyclic trimer, tetramer, and pentamer porphyrins were formed with large association constants through high fidelity selfsorting assembling of corresponding meso-meso-linked diporphyrins. Meso-cinchomeronimide substituents were fully distinguished on the basis of enantiomeric and conformational differences. These studies of non-covalently linked meso-conjugated cyclic porphyrin arrays facilitated the basic understanding of structural features required for such fast and efficient electron and energy transfer processes in natural light harvesting complexes.

\section{Covalent meso linked cyclic porphyrin arrays}

It is established that the effective electronic coupling between neighboring pigments and the regular structural arrangement are highly required for efficient EET. Porphyrin architectures with direct linkage at meso positions comprise an attractive target in terms of higher molecular symmetry, synthetic challenge, and large electronic interactions between neighboring porphyrins that results in efficient EET [78]. In this regard, 5,10-diaryl zinc porphyrin monomer 14 was used as starting material and made to undergo $\mathrm{Ag}(\mathrm{I})$-salt oxidation to synthesize dimer 15 in addition of small amount of trimer 16. Tetramer 17 was also obtained with subsequent reaction of dimer under similar oxidation reaction conditions and hence, long porphyrin arrays with direct linkage at meso positions have been synthesized in this manner (Scheme 2). Intramolecular coupling reaction of these linear porphyrin arrays including trimer, tetramer have been resulted into synthesis of cyclic porphyrin arrays. These cyclic arrays were purified using silicagel column chromatography, and their structures were noticed in accordance with their $1 \mathrm{H}$ NMR spectra, which are characteristically simple without meso-proton signals, reflecting the symmetric cyclic structures [98].

Directly meso-meso linked cyclic porphyrin arrays have been known to exhibit a broad Soret band with red shift, in contrast to their linear meso-meso linked porphyrin array counterpart. An excitonically allowed state of the same energy has been shown in cyclic arrays, as both transition dipole moments $\mathrm{Bx}$ and By of porphyrins are coupled excitonically with their neighboring units. As described above, the $J$-type exciton coupling was exhibited along the long molecular axis in the linear meso-meso linked porphyrin arrays, but $H$-type coupling was also noticed for cyclic tetramer CZ4 and octamer CZ4. This was probably due to deviation of dihedral angles from $90^{\circ}$ in the neighboring porphyrin rings, when the array is bent. The EET rates in all these cyclic porphyrin arrays were determined by the transient absorption anisotropy (TAA) and the transient absorption (TA) measurements. Transient absorption measurements have shown the singlet-singlet excitation annihilation process due to pump power dependent decay process with Forster-type incoherent electron and energy transfer within the porphyrin arrays. Unlike natural cyclic antenna system with B850, directly linked cyclic arrays have shown quite efficient EET processes with the rate constants of $(119 \mathrm{fs})^{-1}$ for CZ4, $(342 \mathrm{fs})^{-1}$ for CZ6, and (236 $\mathrm{fs})^{-1}$ for CZ8. This was explained in terms of extremely strong excitonic coupling between different components of the cyclic porphyrin arrays. The calculated dihedral angles between neighboring porphyrins and their absorption spectra have shown that large electronic coupling between them are of the same order as that of observed order of EET rates with CZ4 at highest side and CZ6 at the lowest one. Pd-catalyzed coupling reaction also gave similar kind of porphyrin octamer with meso linkage [99]. This approach is valuable for the synthesis of hybrid porphyrin arrays with unique structural and configurational features.

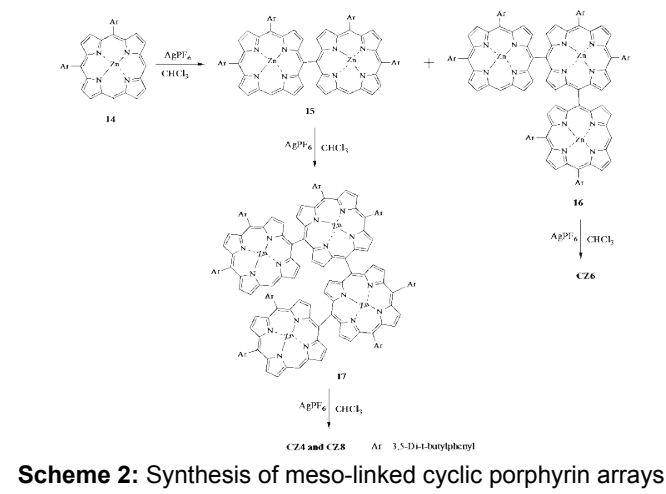

\section{Meso linked diporphyrins to porphyrin boxes}

An angle between the ligand and the porphyrin symmetry plays a key role in the construction of desired molecular assembly. Selfassembly properties can be changed dramatically by changing the geometry of monomer unit of multiporphyrins. Osuka et al. utilized this property in designing the three-dimensional porphyrin boxes. Racemic 4-pyridine-substituted meso-linked $\mathrm{Zn}$ (II) diporphyrins were taken as starting substrates to synthesize the porphyrinic boxes via self-sorting assembling process. Dihedral angle between the 4-pyridyl group and 
the diporphyrin was established at $90^{\circ}$. It has been shown that dihedral angle provides an important contribution during the synthesis of box-shaped porphyrin assemblies. Non-coordinating solvents such as chloroform or benzene have been used to synthesize cyclic porphyrin tetramer 19 from the monomer 5-p-pyridyl-15-(3,5-dioctyloxyphenyl) zinc(II) porphyrin 18 (Scheme 3 ).

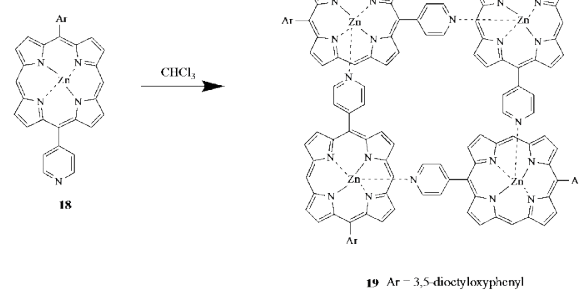

Scheme 3: Synthesis of porphyrin tetramer.

Similarly, a series of 5-p-pyridyl-15-(3,5-dioctyloxyphenyl) zinc(II) porphyrin squares were reported to synthesize and their structures have been confirmed by X-ray crystal structure analysis. The cyclic porphyrin tetramer 19 was characterized by using $1 \mathrm{H}$ NMR and UV-vis absorption spectral analysis [100]. Porphyrin monomer 18 and other analogues have also utilized for $\mathrm{Ag}(\mathrm{I})$-promoted coupling reaction to produce meso coupled diporphyrins in good yields respectively along with higher oligomers. Further, porphyrin dimer has been shown to undergo aggregation to produce a rigid tetrameric conformation with a larger association constant which was characterized by using cold spray ionization mass spectroscopy (CSI-MS) and 1H NMR spectroscopy. The formation of rigid self-assembled structure was further confirmed by fluorescence spectroscopy. On the basis of similar pattern, porphyrin boxes have been produced upon aggregation in solution, which were characterized by $1 \mathrm{H}$ NMR spectroscopy [101].

\section{Conclusion}

In summary, synthesis and spectral properties of meso linked multiporphyrins are reviewed. It was noticed that porphyrins having free meso positions are important precursors for the formation of diporphyrins and other higher oligomers with different shapes including linear, cyclic and boxes and hence, act as an efficient model for light harvesting systems. Study of efficient electron and energy transfer processes in various covalently and non-covalently linked meso-conjugated porphyrin arrays provided an insight view of mimics of light harvesting complexes.

\section{Acknowledgements}

Authors gratefully acknowledge the Council of Scientific and Industrial Research (CSIR) to carry out this work.

\section{References}

1. Sundstrom V, Pullerits T, Grondelle RV (1999) Photosynthetic light-harvesting: reconciling dynamics and structure of purple bacterial LH2 reveals function of photosynthetic unit. J Phys Chem B 103: 2327-2346.

2. Papiz MZ, Prince SM, Howard T, Cogdell RJ, Isaacs NW (2003) The structure and thermal motion of the B800-850 LH2 complex from Rps. acidophila at $2.0 \AA$ resolution and $100 \mathrm{~K}$ : New structural features and functionally relevant motions. J Mol Biol 326: 1523-1538.

3. Cogdell RJ, Isaacs NW, Freer AA, Howard TD, Gardiner AT, et al. (2003) The structural basis of light harvesting in purple bacteria. FEBS Lett 555: 35-39.

4. Bahatyrova S, Frese RN, Siebert CA, Olsen JD, Van der Werf KO, et al. (2004) The native architecture of a photosynthetic membrane. Nature 430: 1058-1062.
5. Kobuke $Y$ (2006) Artificial light-harvesting systems by use of metal coordination Eur J Inorg Chem 2333-2351.

6. Shreve AP, Trautman JK, Frank HA, Owens TG, Albrecht AC (1991) Femtosecond energy-transfer processes in the B800-850 light-harvesting complex of Rhodobacter sphaeroides. Biochim Biophys Acta 1058: 280-288.

7. Jimenez R, Dikshit SN, Bradforth SE, Fleming GR (1996) Electronic excitation transfer in the LH2 complex of Rhodobacter sphaeroides. J Phys Chem 100: 6825-6834.

8. Hu X, Ritz T, Damjanovic A, Schulten K (1997) Pigment organization and transfer of electronic excitation in the photosynthetic unit of purple bacteria. $J$ Phys Chem B 101: 3854-3871.

9. Jungas C, Ranck JL, Rigaud JL, Joliot P, Vermeglio A (1999) Supramolecula organization of the photosynthetic apparatus of Rhodobacter sphaeroides. EMBO J 18: 534-542.

10. Roszak AW, Haward TD, Southall J, Gardiner AT, Law CJ, et al. (2003) Crystal structure of the RC-LH1 core complex from Rhodopseudomonas palustris. Science 302: 1969-1972.

11. Elemans JAAW, Hameren RV, Nolte RJM, Rowan AE (2006) Molecular materials by self-assembly of porphyrins, phthalocyanines and perylenes. Adv Mater 18: 1251-1266.

12. Satake A, Kobuke Y (2005) Dynamic supramolecular porphyrin systems Tetrahedron 61: 13-41

13. Burrell AK, Officer DL, Plieger PG, Reid DCW (2001) Synthetic routes to multiporphyrin arrays. Chem Rev 101: 2751-2796

14. Choi MS, Yamazaki T, Yamazaki I, Aida T (2004) Bioinspired molecular design of light-harvesting multiporphyrin arrays. Angew Chem Int Ed 43: 150-158.

15. Furutsu D, Satake A, Kobuke Y (2005) A Giant Supramolecular Light-Harvesting Antenna-Acceptor Composite. Inorg Chem 44: 4460-4462.

16. Tsuda A, Osuka A (2001) Fully Conjugated Porphyrin Tapes with Electronic Absorption Bands That Reach into Infrared. Science 293: 79-82.

17. Anderson HL (1999) Building molecular wires from the colours of life: conjugated porphyrin oligomers. Chem Commun 2323-2330.

18. Imahori H (2004) Giant Multiporphyrin Arrays as Artificial Light-Harvesting Antennas. J Phys Chem B 108: 6130-6143.

19. Hasobe T, Imahori H, Kamat PV, Ahn TK, Kim SK, et al. (2005) Photovoltaic cells using composite nanoclusters of porphyrins and fullerenes with gold nanoparticles. J Am Chem Soc 127: 1216-1228.

20. Priyadarshy S, Therien MJ, Beratan DN (1996) Acetylenyl-linked, porphyrinbridged, donor-acceptor molecules: a theoretical analysis of the molecular first hyperpolarizability in highly conjugated push-pull chromophore Structures. J Am Chem Soc 118: 1504-1510.

21. Beljonne D, O'Keefe GE, Hamer PJ, Friend RH, Anderson HL, et al. (1997) Investigation of the linear and nonlinear optical response of edge-linked conjugated zinc porphyrin oligomers by optical spectroscopy and configuration interaction techniques. J Chem Phys 106: 9439-9460.

22. Screen TEO, Thorne JRG, Denning RG, Bucknall DG, Anderson HL (2003) Two methods for amplifying the optical nonlinearity of a conjugated porphyrin polymer: transmetallation and self-assembly. J Mater Chem 13: 2796-2808.

23. LeCours SM, Guan HW, DiMagno SG, Wang CH, Therien MJ (1996) Push-pull arylethynyl porphyrins: new chromophores that exhibit large molecular first-order hyperpolarizabilities. J Am Chem Soc 118: 1497-1503.

24. Kessel D, Dougherty TJ, Chang CK (1991) Photosensitization by synthetic diporphyrins and dichlorins in vivo and in vitro. Photochem Photobiol 53: 475479.

25. Hsu CP (2009) The electronic couplings in electron transfer and excitation energy transfer. Acc Chem Res 42: 509-518.

26. Cho HS, Rhee H, Song JK, Min CK, Takase M, et al. (2003) Excitation energy transport processes of porphyrin monomer, dimer, cyclic trimer, and hexamer probed by ultrafast fluorescence anisotropy decay. J Am Chem Soc 125: 58495860 .

27. Paolesse R, Jaquinod L, Sala FD, Nurco DJ, Prodi L, et al. (2000) $\beta$-Fused oligoporphyrins: a novel approach to a new type of extended aromatic system. J Am Chem Soc 122: 11295-11302.

28. Flamigni L, Talarico AM, Chambron JC, Heitz V, Linke M, et al. (2004) 
Photoinduced electron transfer in multiporphyrinic interlocked structures: the effect of copper(i) coordination in the central site. Chem Eur J 10: 2689-2699.

29. Fan J, Whiteford JA, Olenyuk B, Levin MD, Stang PJ, Fleischer EB (1999) Selfassembly of porphyrin arrays via coordination to transition metal bisphosphine complexes and the unique spectral properties of the product metallacyclic ensembles. J Am Chem Soc 121: 2741-2752.

30. Redman JE, Feeder N, Teat SJ, Sanders JKM (2001) Rh(III) Porphyrins as building blocks for porphyrin coordination arrays: from dimers to heterometallic undecamers. Inorg Chem 40: 2486-2499.

31. Sessler JL, Wang B, Harriman A (1995) Photoinduced energy transfer in associated, but noncovalently-linked photosynthetic model systems. J Am Chem Soc 117: 704-714.

32. Ikeda C, Yoon ZS, Park M, Inoue H, Kim D, et al. (2005) Helicity Induction and Two-photon absorbance enhancement in zinc(II) meso-meso linked porphyrin oligomers via intermolecular hydrogen bonding interactions. J Am Chem Soc 127: 534-535.

33. Shirakawa M, Kawano SI, Fujita N, Sada K, Shinkai S (2003) Hydrogenbondassisted control of $h$ versus $j$ aggregation mode of porphyrins stacks in an organogel system. J Org Chem 68: 5037-5044.

34. Holten D, Bocian DF, Lindsey JS (2002) Probing electronic communication in covalently linked multiporphyrin arrays. A guide to the rational design of molecular photonic devices. Acc Chem Res 35: 57-69.

35. Kim D, Osuka A (2004) Directly linked porphyrin arrays with tunable excitonic interactions. Acc. Chem Res 37: 735-745.

36. Lin VSY, Therien MJ (1995) The role of porphyrin-to-porphyrin linkage topology in the extensive modulation of the absorptive and emissive properties of a series of ethynyl- and butadiynyl-bridged bis- and tris(porphinato)zinc chromophores. Chem Eur J 1: 645-651.

37. Ostrowski JC, Susumu K, Robinson MR, Therien MJ, Bazan GC (2003) Nearinfrared electroluminescent light-emitting devices based on ethyne-bridged porphyrin fluorophores. Adv Mater 15: 1296-1300.

38. Anderson HL, Martin DD, Bradley C (1994) Synthesis and third-order nonlinea optical properties of a conjugated porphyrin polymer. Angew Chem Int Ed Engl 33: $655-657$

39. Osuka A, Tanabe N, Kawabata S, Yamazaki I, Nishimura Y (1996) Synthesis and Intramolecular Electron- and Energy-Transfer Reactions of Polyyne- or Polyene-Bridged Diporphyrins. J Org Chem 60: 7177-7185.

40. Nakamura K, Fujimoto T, Takara S, Sugiura KI, Miyasaka H, et al. (2003) Systematic synthesis of porphyrin dimers linked by conjugated oligoacetylene bridges. Chem Lett 32: 694-695.

41. Chachisvilis M, Chirvony VS, Shulga AM, Kallebring B, Larsson S, et al (1996) Spectral and photophysical properties of ethylene-bridged side-toside porphyrin dimers. 1. ground-state absorption and fluorescence study and calculation of electronic structure of trans-1,2-bis(meso-octaethylporphyrinyl) ethene. J Phys Chem 100: 13857-13866.

42. Osuka A, Maruyama K, Mataga N, Asahi T, Yamazaki I, et al. (1990) Geometry dependence of intramolecular photoinduced electron transfer in synthetic zincferric hybrid diporphyrins. J Am Chem Soc 112: 4958-4959.

43. Borowiec J, Trojnar I, Wołowiec S (2004) Mono- and dizinc complexes of diporphyrins with flexible spacers of variable length. Polyhedron 23: 33-39.

44. Mongin O, Papamicael C, Hoyler N, Gossauer A (1998) Modular Synthesis of Benzene-Centered Porphyrin Trimers and a Dendritic Porphyrin Hexamer. J Org Chem 63: 5568-5580.

45. Yu L, Lindsey JS (2001) Investigation of two rational routes for preparing p-phenylene linked porphyrin trimers. Tetrahedron 57: 9285-9298.

46. Thamyongkit P, Lindsey JS (2004) Synthesis of swallowtail-substituted multiporphyrin rods. J Org Chem 69: 5796-5799.

47. Ambroise A, Wagner RW, Rao PD, Riggs JA, Hascoat P, et al. (2001) Design and synthesis of porphyrin-based optoelectronic gates. Chem Mater 13: 1023-1034.

48. Kodis G, Liddell PA, Garza Ldela, Clausen PC, Lindsey JS, et al. (2002) Efficient energy transfer and electron transfer in an artificial photosynthetic antenna-reaction center complex. J Phys Chem A 106: 2036-2048.

49. Oijen AMV, Ketelaars M, Kohler J, Aartsma TJ, Schmidt J (1999) Unraveling the electronic structure of individual photosynthetic pigment-protein complexes. Science 285: 400-402
50. Biemans HAM, Rowan AE, Verhoeven A, Vanoppen P, Latterini L, et al. (1998) Hexakis porphyrinato benzenes. a new class of porphyrin arrays $\mathrm{J}$ Am Chem Soc 120: 11054-11060

51. Chichak K, Brand NR (2000) The metal-directed self-assembly of threedimensional porphyrin arrays. Chem Comm 13: 1211-1212.

52. Takahashi R, Kobuke $Y$ (2003) hexameric macroring of gable-porphyrins as a light-harvesting antenna mimic. J Am Chem Soc 125: 2372-2373.

53. Seth J, Palaniappan V, Johnson TE, Prathapan S, Lindsey JS, et al. (1994) investigation of electronic communication in multi-porphyrin light-harvesting arrays. J Am Chem Soc 116: 10578-10592.

54. SolladiL N, Gross M, Gisselbrecht JP, Sooambar C (2001) Pentaporphyrin with flexible, chiral nucleosidic linkers: unexpected duality of the physico-chemical properties of its core. Chem Comm 2206-2207.

55. Nakano A, Osuka A, Yamazaki I, Yamazaki T, Nishimura Y (1998) Windmill-like porphyrin arrays as potent light-harvesting antenna complexes. Angew Chem Int Ed 37: 3023-3027.

56. Nakano A, Yamazaki T, Nishimura Y, Yamazaki I, Osuka A (2000) Threedimensionally arranged windmill and grid porphyrin arrays by agi-promoted meso-meso block oligomerization. Chem Eur J 6: 3254-3271.

57. Mak CC, Pomeranc D, Montalti M, Prodi L, Sanders JMK (1999) A versatile synthetic strategy for construction of large oligomers: binding and photophysical properties of a nine-porphyrin array. Chem Comm 1083-1084.

58. Kasha M, Rawls HR, El-Bayoumi MA (1965) The exciton model in molecular spectroscopy. Pure Appl Chem 11: 371-392.

59. Mongin O, Hoyler N, Gossauer A (2000) Synthesis and light-harvesting properties of niphaphyrins, Eur J Org Chem 1193-1197.

60. Rucareanu S, Mongin O, Schuwey A, Hoyler N, Gossauer A, et al. (2001) Supramolecular assemblies between macrocyclic porphyrin hexamers and star-shaped porphyrin arrays. J Org Chem 66: 4973-4988.

61. Kato A, Sugiura K, Miyasaka H, Tanaka H, Kawai T, et al. (2004) A square cyclic dodecamer: Synthesis and single-molecule characterization. Chem Lett 33: 578 .

62. Wasielewski MR (1992) Photoinduced electron transfer in supramolecular systems for artificial photosynthesis. Chem Rev 92: 435-461.

63. Aratani N, Osuka A, Cho HS, Kim D (2002) Photochemistry of covalently-linked multi-porphyrinic systems. J Photochem Photobiol C: Photochem Rev 3: 25-52.

64. Hwang IW, Aratani N, Osuka A, Kim D (2005) Excitation energy migration in multiporphyrin arrays. Bull Korean Chem Soc 26: 19-31.

65. Kim D, Osuka A (2004) Directly linked porphyrin arrays with tunable excitonic interactions. Acc Chem Res 37: 735-745.

66. Susumu K, Shimidzu T, Tanaka K, Segawa H (1996) Synthesis of nove porphyrin arrays directly-linked through the meso-carbons. Tetrahedron Lett 37: 8399-8402.

67. Khoury RG, Jaquinod L, Smith KM (1997) Rational approach to the synthesis of meso-meso (5,5 linked bis-porphyrins. Chem Commun 1057-1058.

68. Osuka A, Shimidzu H (1997) Meso-meso linked porphyrin arrays. Angew Chem Int Ed Eng 36: 135-137.

69. Ogawa T, Nishimoto Y, Yoshida N, Ono N, Osuka A, et al. (1998) One-pot electrochemical formation of meso-meso linked porphyrin arrays Chem Comm 337-338.

70. Senge MO, Feng X (1999) Synthesis of directly meso-meso linked bisporphyrins using organolithium reagents. Tetrahedron Lett 40: 4165-4168.

71. Shi X, Amin SR, Liebeskind LS (2000) 3-Cyclobutenyl-1,2-dione-substituted Porphyrins. 2. A Simple and general entry to quinone-porphyrin-porphyrinquinon tetrads and related molecules. J Org Chem 65: 1665-1671.

72. Osuka A (2015) Towards meso-meso linked porphyrin arrays and meso ary expanded porphyrins. Chem Rec 15: 143-159.

73. Yang J, Yoo H, Aratani N, Osuka A, Kim D (2009) Determination of the superradiance coherence length of directly linked linear porphyrin arrays at the single-molecule level. Angew Chem Int Ed 48: 4323-4327.

74. Nakamura Y, Hwang IW, Aratani N, Ahn TK, Ko DM, et al. (2005) Directly meso-meso linked porphyrin rings: Synthesis, characterization, and efficient excitation energy hopping. J Am Chem Soc 127: 236-246. 
75. Jin LM, Chen L, Yin JJ, Guo CC, Chen QY (2005) A facile and potent synthesis of meso,meso-linked porphyrin arrays using iodine(III) reagents. Eur J Org Chem 3994-4001.

76. Ikeda T, Aratani N, Easwaramoorthi S, Kim D, Osuka A (2009) Meso- $\beta$ Doubly Linked Zn(II) Porphyrin Trimers: Distinct anti-versus-syn Effects on Their Photophysical Properties. Org Lett 11: 3080-3083.

77. Yoon MC, Noh SB, Tsuda A, Nakamura Y, Osuka A, et al. (2007) Photophysics of meso-; doubly linked Ni(II) porphyrin arrays: Large twophoton absorption cross-section and fast energy relaxation dynamics. J Am Chem Soc 129 10080-10081.

78. Mori H, Tanaka T, Lee S, Lim JM, Kim D, et al. (2015) Meso-meso linked porphyrin-[26]hexaphyrin-porphyrin hybrid arrays and their triply linked tapstrong absorp;tion bands in NIR region. J Am Chem Soc 137: 20197-2106.

79. Anderson HL, Wylie AP, Prout K (1998) Meso-tetraalkynylporphyrins. J Chem Soc Perkin Trans 1: 1607-1611.

80. Anderson HL, Martin SJ, Bradley DDC (2016) Chemistry of meso-aryl substituted expanded porphyrins: Aromaticity and molecular twist. Chem Rev 117: $2584-2640$

81. Balaz M, Collins HA, Dahlstedt E, Anderson HL (2009) Synthesis of hydrophilic conjugated porphyrin dimers for one-photon and two-photon photodynamic therapy at NIR wavelengths. Org Biomol Chem 7: 874-888.

82. Kuimova MK, Collins HA, Balaz M, Dahlstedt E, Levitt JA, et al. (2009) Photophysical properties and intracellular imaging of water-soluble porphyrin dimers for two-photon excited photodynamic therapy. Org Biomol Chem 7: 889896

83. Richardson C, Reed CA (2007) Synthesis of meso-extended tetraaryl porphyrins. J Org Chem 72: 4750-4755.

84. Rai S, Ravikanth M (2008) Synthesis of covalently linked unsymmetrical porphyrin pentads containing three different porphyrin subunits. J Org Chem 73: $8364-8375$

85. Shivran N, Gadekar SC, Anand VG (2017) To twist or not to twist: Figure-ofeight and planar structures of octaporphyrins. Chem Asian J 12: 6-20.

86. Patten PGV, Shreve AP, Lindsey JS, Donhoe RJ (1998) Energy-transfer modeling for the rational design of multiporphyrin light-harvesting arrays. J Phys Chem B 102: 4209-4216.

87. Arnold DP, Hartnell RD (2001) Butadiyne-linked bis(chlorin) and chlorinporphyrin dyads and an improved synthesis of bis[octaethylporphyrinatonicke I(II)-5-yl]butadiyne using the Takai iodoalkenation Tetrahedron 57: 1335-1345.

88. Gros CP, Brisach F, Meristoudi A, Espinosa E, Guilard R, et al. (2007) Modulation of the singlet-singlet through-space energy transfer rates in cofacial bisporphyrin and porphyrin-corrole dyads. Inorg Chem 46: 125-135.
89. Shinoda S, Tsukube H, Nishimura Y, Yamazaki I, Osuka A (1997) Energy and electron transfer properties of methyl pheophorbide-a in zinc porphyrinpheophorbide dyads. Tetrahedron 53: 13657-13666.

90. Yang SI, Li J, Cho HS, Kim D, Bocian DF, et al. (2000) Synthesis and excitedstate photodynamics of phenylethyne-linked porphyrin-phthalocyanine dyads. J Mater Chem 10: 283-296.

91. Cho HS, Jeong DH, Yoon MC, Kim YH, Kim YR, et al. (2001) Excited-state energy transfer processes in phenylene- and biphenylene-linked and directlylinked zinc(II) and free-base hybrid diporphyrins. J Phys Chem A 105: 42004210.

92. Cho HS, Song NW, Kim YH, Jeoung SC, Hahn S, et al. (2000) Ultrafast energy relaxation dynamics of directly linked porphyrin arrays. J Phys Chem A 104: 3287-3298.

93. Yoshida N, Osuka A (2000) Control of dihedral angle of meso-meso linked diporphyrins by introducing dioxymethylene straps of various length. Org Lett 2: 2963-2966.

94. Yoshida N, Jeong DH, Cho HS, Kim D, Matsuzaki Y, et al. (2003) Fine tuning of photophysical properties of meso-meso-linked Znll-diporphyrins by dihedral angle control. Chem Eur J 9: 58-75.

95. Jeong DH, Jang SM, Hwang IW, Kim D, Yoshida N, et al. (2002) Investigation of interporphyrin charge resonance of dihedral angle controlled porphyrin dimers by resonance raman spectroscopy and MO approaches. J Phys Chem A 106: 11054-11063.

96. Cho HS, Song JK, Ha JH, Cho S, Kim D, et al. (2003) Comparative studies on energy relaxation dynamics of directly linked $\mathrm{Zn}$ " porphyrin dimers with different dihedral angles. J Phys Chem A 107: 1897-1903.

97. Martin RE, Diederich F (1999) Linear monodisperse conjugated oligomers: mode compounds for polymers and more. Angew Chem Int Ed Engl 38: 1350-1377.

98. Aratani N, Kim D, Osuka A (2009) Discrete cyclic porphyrin arrays as artificia light-harvesting antenna. Accounts of Chemical Research 42: 1922-1934.

99. Aratani N, Osuka A (2008) A meso-meso directly linked octameric porphyrin square. Chem Commun 4067-4069.

100. Tsuda A, Nakamura T, Sakamoto S, Yamaguchi K, Osuka A (2002) A SelfAssembled Porphyrin Box from meso-meso-Linked Bis\{5-p-pyridyl-15-(3,5di-octyloxyphenyl)porphyrinato zinc(II)\}. Angew Chem Int Ed 41: 2817-2821.

101. Hwang IW, Kamada T, Ahn TK, Ko DM, Nakamura T, et al. (2004) Porphyrin Boxes Constructed by Homochiral Self-Sorting Assembly: Optical Separation, Exciton Coupling, and Efficient Excitation Energy Migration. J Am Chem Soc 126: $16187-16198$ 